\title{
ENFERMEDADES TRANSMISIBLES, SALUD MENTAL Y EXPOSICIÓN A CONTAMINANTES AMBIENTALES EN POBLACIÓN ALEDAÑA AL PROYECTO MINERO LAS BAMBAS ANTES DE LA FASE DE EXPLOTACIÓN, PERÚ 2006
}

\author{
Jonh Astete ${ }^{1, a}$, María del Carmen Gastañaga $a^{1, a}, V_{1}$ ctor Fiestas $^{2, a}$, Tania Oblitas ${ }^{1, b}$, Iselle Sabastizagal ${ }^{1, c}$, \\ Martha Lucero ${ }^{1, \mathrm{c}}$, Jesús del Milagro Abadíe ${ }^{1, \mathrm{~d}}$, María Elena Muñoz ${ }^{2, \mathrm{e}}$, Ada Valverde $^{2, \mathrm{e}}$, Magna Suarez $^{2, \mathrm{f}}$
}

\begin{abstract}
RESUMEN
Objetivo. Determinar la prevalencia de enfermedades transmisibles, salud mental y exposición a contaminantes ambientales en la población aledaña al proyecto minero Las Bambas antes de la fase de explotación. Materiales $y$ métodos. Estudio descriptivo transversal realizado en 453 personas (niños y adultos) residentes en tres distritos de la región Apurímac: Haquira, Chalhuahuacho y Progreso. Se evaluó el desarrollo psicomotor, coeficiente intelectual y niveles de ansiedad y depresión, así como la presencia de enfermedades transmisibles (sífilis, VIH, hepatitis viral B, C y Delta,) metales pesados (nivel de plomo en sangre, nivel de cadmio, arsénico y mercurio, en orina) y colinesterasa sérica. Resultados. La edad promedio fue de 29,0 $\pm 17,3$ años, 59,2\% fueron mujeres y el tiempo de residencia fue de 6 a 15 años. No se encontraron casos de $\mathrm{VIH}$, hepatitis $\mathrm{C}$ y Delta, $1,4 \%$ fueron positivos a sífilis y en relación con hepatitis $\mathrm{B}$ se encontró $1,7 \%$ positivos a anti $\mathrm{HBc}$ total y $0,5 \%$ positivos a $\mathrm{HBs}$ Ag. Se encontraron valores por encima de lo permitido de mercurio en $1,8 \%$ de la población, arsénico en $4,6 \%$, plomo en $24,3 \%$ y cadmio en $43,9 \%$. Además, el $29,1 \%$ de la población tuvo valores de colinesterasa inferiores al rango referencial. En la población infantil, 12,5\% presentaba riesgo en su desarrollo psicomotor; $2,1 \%$ y $3,1 \%$ presentaba retardo mental leve y fronterizo, respectivamente; $34,3 \%$ de los mayores de 12 años sufrían de ansiedad y 17,5\% de depresión. Conclusiones. Existen evidencias de contaminación ambiental por metales pesados y presencia de enfermedades transmisibles en esta población. Una futura explotación minera irresponsable podría agravar dicha situación epidemiológica.
\end{abstract}

Palabras clave: Minería; Enfermedades transmisibles; Plaguicidas; Contaminantes ambientales; Salud mental; Asentamientos rurales; Perú (fuente: DeCs BIREME).

\section{COMUNICABLE DISEASES, MENTAL HEALTH AND EXPOSURE TO ENVIRONMENTAL POLLUTANTS IN POPULATION LIVING NEAR LAS BAMBAS MINING PROJECT BEFORE EXPLOITATION PHASE, PERU 2006}

\begin{abstract}
Objective. To determine the prevalence of communicable diseases, mental health and environmental pollutants exposure in population living near Las Bambas mining project before exploitation phase. Material and methods. Cross sectional study performed in 453 subjects (children and adults) living in three Apurimac region districts: Haquira, Chalhuahuacho and Progreso. Psychomotor development, intelligence quotient, anxiety and depression levels and the presence of communicable diseases (viral hepatitis B, C and delta, syphilis and HIV) were evaluated, as well as heavy metals (lead in blood, and cadmium, arsenic and mercury in urine samples) and serum cholinesterase levels. Results. Mean age was 29 \pm 17.25 years, $59.2 \%$ were female and a range of 6 to 15 years of living in the area was found. No cases of HIV, hepatitis $\mathrm{C}$ and delta were found, $1.4 \%$ were positive for syphilis and in relation to hepatitis $\mathrm{B}$, we found $1,7 \%$ of subjects positive to total anti $\mathrm{HBc}$ and $0.5 \%$ positive for HBsAg. Heavy metal testing identified people with exceeding limits of mercury in $1.8 \%$ arsenic in $4.6 \%$, lead in $24.3 \%$ and cadmium in $43.9 \%$. Besides, $29.1 \%$ of the population had cholinesterase levels below normal range. Among children, $12.5 \%$ were at psychomotor development levels of risk; $2.1 \%$ and $3.1 \%$ suffered from mild and borderline intellectual disability (mental retardation), respectively. $34.3 \%$ of subjects older than 12 had anxiety and $17.5 \%$ depression. Conclusions. Evidence of heavy metal environmental pollution and presence of communicable diseases in this population were already found. Future careless mining activity could worsen the current health situation.
\end{abstract}

Key words: Health profile; Mining; Communicable diseases; Pesticides; Environmental pollutants; Mental health; Rural settlements; Peru (source: MeSH NLM).

\footnotetext{
Centro Nacional de Salud Ocupacional y Protección del Ambiente para la Salud, Instituto Nacional de Salud. Lima, Perú.

Centro Nacional de Salud Pública, Instituto Nacional de Salud. Lima, Perú.

Médico; ${ }^{b}$ Enfermera; ${ }^{c}$ Psicóloga; d Técnico de Laboratorio; ${ }^{e}$ Tecnólogo Médico; ${ }^{\text {f Bióloga. }}$
}

Recibido: 09-08-10 Aprobado: 01-12-10 


\section{INTRODUCCIÓN}

El Perú es uno de los siete países mineros más ricos del orbe y la minería sigue siendo uno de los pilares de nuestra economía, representando anualmente 1,6 billones de dólares en exportaciones. En la década pasada el $49 \%$ del total de productos de exportación correspondieron a minería, constituyéndose en una fuente importante de divisas que impulsa el desarrollo alrededor del área de influencia donde se ubica la operación.

En octubre del año 2004, el estado peruano suscribió un contrato de concesión con la empresa suiza Xstrata Cooper (1), ganadora de la licitación internacional, para realizar la etapa de exploración y factibilidad del proyecto minero Las Bambas ${ }^{(2,3)}$, el cual se encuentra ubicado en las provincias de Cotabambas y Grau de la región Apurímac y es considerado uno de los más importantes del país ya que posee yacimientos de cobre, oro, plata y hierro. Este proyecto constituye una gran posibilidad de desarrollo económico y social para la región Apurímac, considerada como de extrema pobreza ${ }^{(4)}$; sin embargo, existe el riesgo de que el desarrollo de la actividad minera ocasione graves consecuencias en el medio ambiente ${ }^{(5,6)}$ y en la salud de las poblaciones residentes dentro del área de influencia, ya sea por un inadecuado manejo de las sustancias contaminantes o por la diseminación de enfermedades infecciosas debido a la utilización de fuerza de trabajo migratoria ${ }^{(7,8)}$, creando así condiciones de vida que incrementan la susceptibilidad a enfermar.

Por ello, se hace necesario establecer la situación inicial del escenario y caracterizar en forma más precisa a la población aledaña al proyecto minero, lo cual servirá como punto de comparación a futuras evaluaciones destinadas ya sea a determinar el alcance de los objetivos o a reformularlos con miras de ganar mayor pertinencia, eficacia, eficiencia y sostenibilidad potencial en el manejo ambiental. El objetivo del estudio fue determinar el perfil epidemiológico de la población aledaña al Proyecto Minero Las Bambas en relación a enfermedades transmisibles, salud mental y por exposición a contaminantes ambientales.

\section{MATERIALES Y MÉTODOS}

\section{DISEÑO Y LUGAR DE ESTUDIO}

Estudio descriptivo de corte transversal realizado en el año 2006 en tres distritos de la región Apurímac: Haquira, Chalhuahuacho y Progreso (Figura 1), que se encuentran en el área de influencia del proyecto minero Las Bambas.

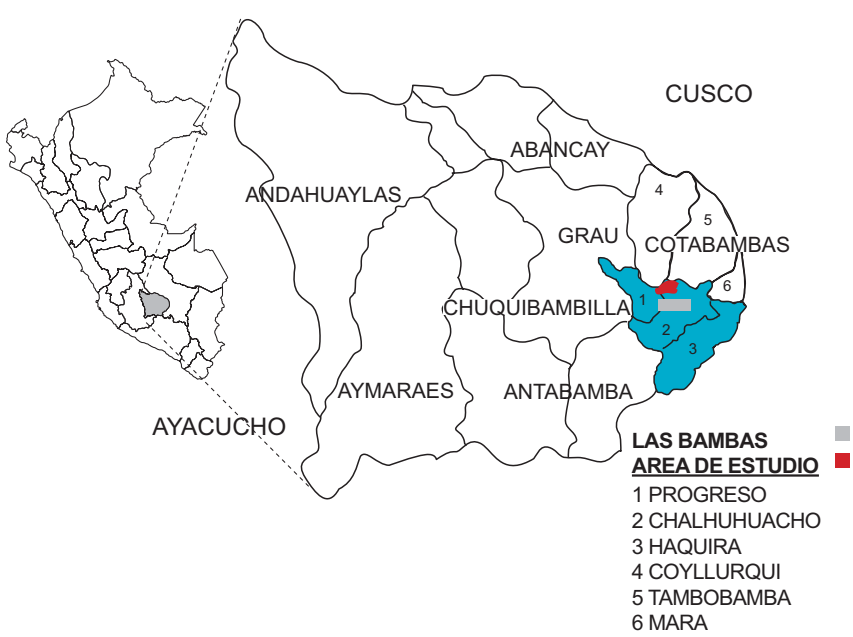

Figura 1. Mapa de provincia de Apurímac donde se desarrolla el Proyecto Minero Las Bambas 2006.

\section{POBLACIÓN Y MUESTRA DEL ESTUDIO}

El estudio tuvo como marco muestral el "Censo Socioeconómico, Demográfico y Epidemiológico-Las Bambas 2006" realizado por el Instituto Nacional de Salud (INS) en los distritos de Haquira, Chalhuahuacho y Progreso de la región Apurímac, en donde se reportó una población de 19821 habitantes.

Se realizó un muestreo aleatorio simple con distribución uniforme teniendo en cuenta una prevalencia máxima a la exposición del $50 \%$, una precisión del $5 \%$ y un nivel de confianza del $95 \%$. Además, se consideró un incremento del $20 \%$ por pérdida o rechazo para asegurar que 377 muestras sean transportadas y procesadas con éxito, obteniendo un tamaño muestral de 453 personas. Se incluyeron a los individuos mayores de un año con un tiempo mínimo de seis meses de residencia en el área de influencia del proyecto Las Bambas, que aceptaron participar y firmaron el consentimiento informado, en el caso de los menores de 18 años se obtuvo la autorización del padre o tutor, según correspondía, así como su asentimiento.

\section{PROCEDIMIENTO E INSTRUMENTOS}

A cada persona que aceptó participar, se le extrajo una muestra de sangre venosa $(7 \mathrm{~mL}$ con EDTA y $3 \mathrm{~mL}$ con heparina) y se solicitó una muestra de orina que fueron transportadas en cadena de frío a los laboratorios del Instituto Nacional de Salud (INS) en Lima para su procesamiento.

En los laboratorios del Centro Nacional de Salud Pública (CNSP) del INS se procesaron las muestras de sangre para determinar marcadores serológicos de 
enfermedades infecciosas $(\mathrm{VIH}$, sífilis, hepatitis $\mathrm{B}, \mathrm{C}$ y Delta) ${ }^{(9)}$, en el laboratorio químico toxicológico del Centro Nacional de Salud Ocupacional y Protección del Ambiente para la Salud (CENSOPAS) del INS se midió la concentración de plomo en sangre y de colinesterasa sérica; así como cadmio, arsénico y mercurio en orina. Se consideró presencia de metales pesados cuando la concentración de plomo fue $>10 \mu \mathrm{g} / \mathrm{dL}$, cadmio $>1 \mu \mathrm{g} / \mathrm{L}$; arsénico $>100 \mu \mathrm{g} / \mathrm{L}$ y mercurio $>5 \mu \mathrm{g} / \mathrm{L}$.

Se evaluó el desarrollo psicomotor de niños menores de tres años usando el TEPSI calificando a los niños en estado normal, riesgo o déficit ${ }^{(10)}$, el coeficiente intelectual se midió a los niños de tres a 12 años con la prueba de Stanford-Binet y fueron clasificados en retardo mental leve, fronterizo, normal bajo, normal, normal superior o superior ${ }^{(11)}$. En mayores de 12 años se evaluó los niveles de ansiedad y depresión con las escalas de Zung y se clasificaron en intensa, leve, moderada, normal o no presenta; finalmente, se midió la violencia familiar con un instrumento que indica el consumo de alcohol del jefe del hogar, maltrato, violencia o no violencia.

\section{ASPECTOS ÉTICOS}

El estudio fue aprobado por el Comité de Ética en Investigación del Instituto Nacional de Salud. Los individuos fueron debidamente informados sobre el propósito de la investigación, siendo la decisión de participar autónoma y respaldada por la firma de un consentimiento informado. Los resultados obtenidos fueron llevados a los establecimientos de salud de la población evaluada para que sean entregados individualmente a cada participante, el cual recibiría consejería e indicaciones por el personal de salud del establecimiento de salud, previamente capacitado por el CENSOPAS/INS.

\section{ANÁLISIS DE DATOS}

Los datos fueron ingresados a una base de Excel y procesados con el paquete estadístico SPSS versión 11. En el análisis descriptivo para variables numéricas se obtuvo medias y desviaciones estándar, Para variables categóricas se obtuvo proporciones y porcentajes.

\section{RESULTADOS}

\section{CARACTERÍSTICAS DE LA POBLACIÓN}

Se incluyeron 453 participantes, 59,2\% fueron mujeres, la edad promedio fue de $29,0 \pm 17,3$ años, $49,2 \%$ tuvieron entre 26 a 60 años. Del total de la muestra,
Tabla 1. Características generales de la población de estudio. Las Bambas 2006

\begin{tabular}{|c|c|c|}
\hline Características & $n=453$ & $(\%)$ \\
\hline \multicolumn{3}{|l|}{ Sexo } \\
\hline Femenino & 268 & $(59,2)$ \\
\hline Masculino & 185 & $(40,8)$ \\
\hline \multicolumn{3}{|c|}{ Rango de edad (años) } \\
\hline$\leq$ a 02 & 4 & $(0,9)$ \\
\hline 02 a 12 & 86 & $(19,0)$ \\
\hline 13 a 18 & 59 & $(13,0)$ \\
\hline 19 a 25 & 56 & $(12,4)$ \\
\hline 26 a 60 & 223 & $(49,2)$ \\
\hline$\geq a 61$ & 25 & $(5,5)$ \\
\hline \multicolumn{3}{|l|}{ Distrito } \\
\hline Haquira & 154 & $(34,0)$ \\
\hline Chalhuahuacho & 214 & $(47,2)$ \\
\hline Progreso & 85 & $(18,8)$ \\
\hline \multicolumn{3}{|c|}{ Tiempo de residencia (años) } \\
\hline$\leq \mathrm{a} 05$ & 70 & $(15,5)$ \\
\hline 05 a 15 & 110 & $(24,3)$ \\
\hline 16 a 25 & 75 & $(16,6)$ \\
\hline 26 a 35 & 75 & $(16,6)$ \\
\hline 36 a 45 & 52 & $(11,5)$ \\
\hline 46 a 65 & 58 & $(12,8)$ \\
\hline$\geq a 66$ & 13 & $(2,9)$ \\
\hline
\end{tabular}

el $24,3 \%$ tuvo un tiempo de residencia de 6 a 15 años; mientras que el $11,5 \%$ nació fuera de las localidades intervenidas. Cabe mencionar que el mayor número de participantes residía en el distrito de Chalhuahuacho (Tabla 1).

\section{ENFERMEDADES TRANSMISIBLES}

Con relación a las enfermedades transmisibles estudiadas (Tabla 2 ) se encontró que para hepatitis $B$ dos personas $(0,5 \%)$ eran portadoras de HBsAg y 31 $(7,1 \%)$ presentaron anticuerpos anticore (anti-HBc), pero en ninguno de ellos se encontró anticuerpos IgM anti-HBc; los casos se presentaron en individuos mayores de 26 años de los distritos de Chalhuahuacho y Haquira. No se encontró portadores para hepatitis $C$ y Delta. En relación con sífilis, la prueba RPR para sífilis resultó reactiva en nueve personas $(2,1 \%)$ y la prueba TPHA confirmó infección por Treponema pallidum en seis $(1,4 \%)$. Finalmente, no se encontró portadores de infección por VIH en la población estudiada. 
Tabla 2. Prevalencia de enfermedades infecciosas en población aledaña al proyecto minero Las Bambas, Apurímac 2006.

\begin{tabular}{|c|c|c|c|c|c|c|c|c|}
\hline \multirow{3}{*}{ Características } & \multicolumn{2}{|c|}{ Anti HBc total } & \multicolumn{2}{|c|}{ HBs Ag } & \multicolumn{2}{|c|}{ RPR } & \multicolumn{2}{|c|}{ TPHA } \\
\hline & Positivo & Negativo & Positivo & Negativo & Positivo & Negativo & Positivo & Negativo \\
\hline & n $(\%)^{*}$ & $n(\%)^{*}$ & n (\%) $)^{*}$ & n $(\%)^{*}$ & n $(\%)^{*}$ & n (\%)* & $n(\%)^{*}$ & n $(\%)^{*}$ \\
\hline \multicolumn{9}{|l|}{ Rango de edad } \\
\hline Menor de 2 años & $0(0,0)$ & $4(0,9)$ & $0(0,0)$ & $4(0,9)$ & $0(0,0)$ & $4(0,9)$ & $0(0.0)$ & $4(0,9)$ \\
\hline $02-12$ años & $0(0,0)$ & $86(18,9)$ & $0(0,0)$ & $86(18,9)$ & $1(0,2)$ & $85(18,8)$ & $0(0,0)$ & $86(18,9)$ \\
\hline $13-18$ años & $1(0,2)$ & $58(12,9)$ & $0(0,0)$ & $59(13,0)$ & $1(0,2)$ & $58(12,9)$ & $0(0,0)$ & $59(13,0)$ \\
\hline $19-25$ años & $3(0,7)$ & $53(11,8)$ & $1(0,2)$ & $55(12,1)$ & $0(0,0)$ & $56(12,4)$ & $0(0,0)$ & $56(12,4)$ \\
\hline $26-60$ años & $23(5,1)$ & $200(44,0)$ & $1(0,2)$ & $222(49,0)$ & $7(1,6)$ & $216(47,8)$ & $6(1,4)$ & $217(47,9)$ \\
\hline 61 años a más & $4(0,9)$ & $21(4,6)$ & $0(0,0)$ & $25(5,5)$ & $0(0,0)$ & $25(5,5)$ & $0(0,0)$ & $25(5,5)$ \\
\hline \multicolumn{9}{|l|}{ Sexo } \\
\hline Femenino & $18(4,1)$ & $250(55,1)$ & $1(0,2)$ & $267(58,9)$ & $5(1,2)$ & $263(58,1)$ & $3(0,7)$ & $265(58,5)$ \\
\hline Masculino & $13(3,0)$ & $172(38,0)$ & $1(0,2)$ & $184(36,4)$ & $4(0,9)$ & $181(40,0)$ & $3(0,7)$ & $182(40,2)$ \\
\hline \multicolumn{9}{|l|}{ Distrito } \\
\hline Haquira & $10(2,2)$ & $144(31,7)$ & $0(0,0)$ & $154(34,0)$ & $5(1,2)$ & $149(33,0)$ & $3(0,7)$ & $151(33,3)$ \\
\hline Chalhuahuacho & $17(3,9)$ & $197(43,6)$ & $2(0,5)$ & $212(46,7)$ & $3(0,7)$ & $211(46,6)$ & $3(0,7)$ & $211(46,6)$ \\
\hline Progreso & $4(0,9)$ & $81(17,8)$ & $0(0,0)$ & 85 (18.8) & $1(0,2)$ & $84(18,3)$ & $0(0,0)$ & $85(18,8)$ \\
\hline
\end{tabular}

*Porcentajes calculados sobre el total de evaluados $(\mathrm{N}=453)$.

\section{EXPOSICIÓN A CONTAMINANTES AMBIENTALES}

Se encontró valores promedio de plomo en sangre en la población evaluada $(11,0 \pm 6,1 \mathrm{ug} / \mathrm{dL}$ con valores máximos de $25,7 \mathrm{ug} / \mathrm{dL}$ ), en el caso de arsénico en orina la media fue de 55,9 $\pm 30,0 \mu \mathrm{g} / \mathrm{L}$ con valores máximos de 201,5 $\mu \mathrm{g} / \mathrm{L}$. En Haquira se encontraron los valores más bajos para ambos metales pesados. Para el cadmio la media poblacional fue de 1,5 $\pm 0,7 \mu \mathrm{g} / \mathrm{L}$ que fue similar en todas las poblaciones, el valor máximo encontrado fue de $3,5 \mu \mathrm{g} / \mathrm{L}$. En relación con el mercurio se encontró un caso con un valor muy alto $(65,1 \mu \mathrm{g} / \mathrm{L})$, la mediana poblacional fue de 1,7 $\pm 0,9 \mu \mathrm{g} / \mathrm{L}$. La colinesterasa sérica tuvo valores promedio de $10454,7 \mathrm{U} / \mathrm{mL}$ con valores más altos en Chalhuahuacho (Figura 2).

Respecto a los contaminantes ambientales, se encontró que el $1,8 \%$ presentó valores por encima de los niveles permitidos de mercurio, $4,6 \%$ de arsénico, $24,3 \%$ de plomo, $43,9 \%$ de cadmio, para el caso de la colinestersa sérica $29,1 \%$ presentaron valores inferiores a lo permitido (Tabla 3).

Se encontró que los varones presentaron con mayor frecuencia niveles elevados de cadmio y plomo. Según distrito, no se encontró casos con niveles elevados de arsénico en Haquira y, en general, fue el distrito que presentó menor proporción de sujetos con valores anormales de los contaminantes ambientales evaluados.
Por otro lado, en Progreso solo $55,2 \%$ de su población presentó valores de plomo menores al límite permitido (Tabla 3). Al evaluar según rango de edad, los individuos más afectados fueron los que se encontraban entre los 26 a 60 años de edad.

\section{PERFIL PSICOLÓGICO}

Al evaluar el nivel de desarrollo psicomotor en los menores de tres años con la prueba TEPSI, se encontró que el $12,5 \%$ presentaba riesgo en su desarrollo psicomotor, mientras que el resto se hallaba normal. En referencia a la evaluación del coeficiente intelectual con la prueba Stanford-Binet en los niños de 3 a 12 años de edad, hubo casos de retardo mental leve $(2,1 \%)$ y retardo mental fronterizo $(3,1 \%)$, pero la mayoría fue normal (50\%) o normal bajo (40,6\%). En cuanto a los niveles de ansiedad y depresión en la población mayor de 12 años, se encontró que el $34,3 \%$ presentaba ansiedad y $17,5 \%$ presentaba depresión.

\section{DISCUSIÓN}

El Perú se encuentra ubicado entre los países de endemicidad intermedia para hepatitis B según lo establecido por la Organización Mundial de la Salud (prevalencia de HBsAg: $2 \%-7 \%$ y Anti HBc: 15\%-40\%), tomando como promedio la prevalencia de marcadores 
(a)

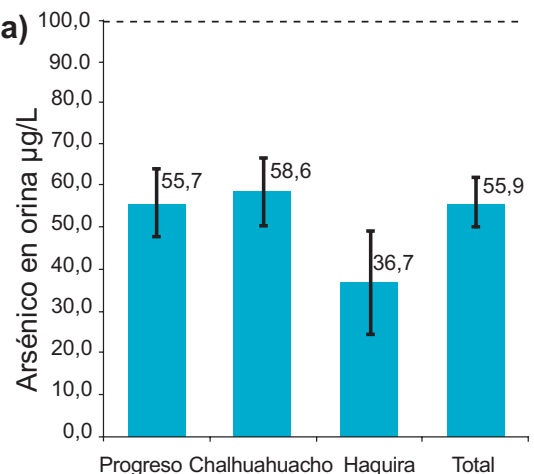

(d)

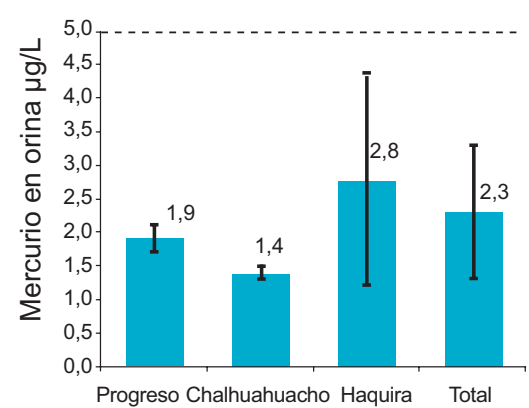

(b)

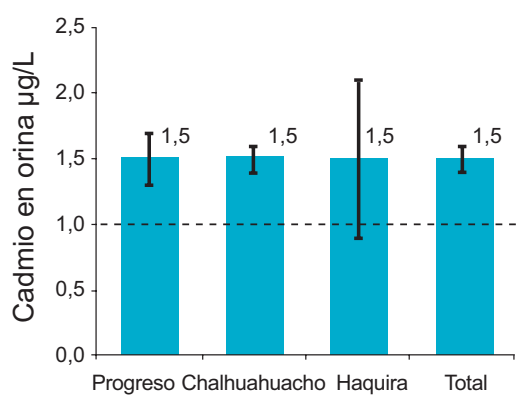

(e)

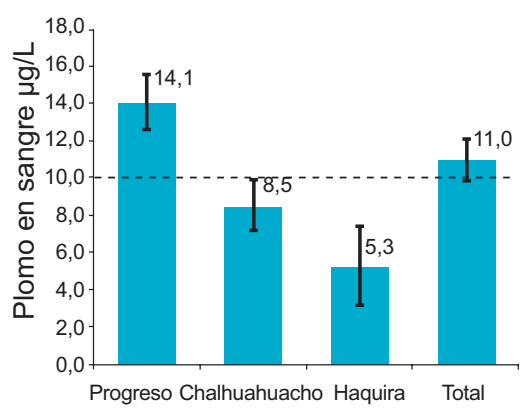

(c)

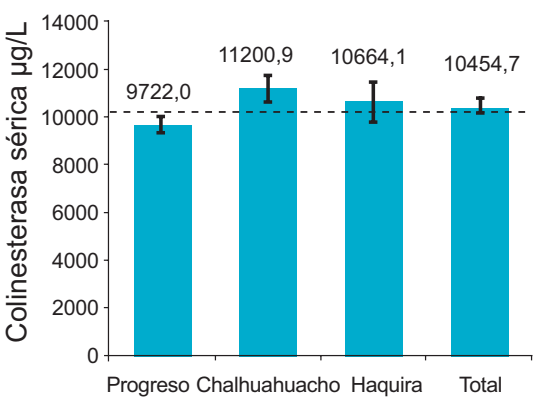

Figura 2. Valores promedio de arsénico en orina (a), cadmio en orina (b), colinesterasa sérica (c), mercurio en orina (d) y plomo en sangre (e) según población aledaña al proyecto minero Las Bambas antes de la fase de explotación, Apurímac, Perú 2006.

Línea demarca el límite para considerar niveles anormales del contaminante ambiental, las barras indican el intervalo de confianza al $95 \%$.

Tabla 3. Niveles anormales de contaminantes ambientales en población aledaña al proyecto minero Las Bambas antes de la fase de explotación, según género y distrito. Apurímac, Perú 2006.

\begin{tabular}{|c|c|c|c|c|c|c|c|c|c|c|c|c|}
\hline \multirow{3}{*}{ Metales pesados } & \multicolumn{4}{|c|}{ Sexo } & \multicolumn{6}{|c|}{ Distrito } & \multirow{2}{*}{\multicolumn{2}{|c|}{ Total }} \\
\hline & \multicolumn{2}{|c|}{ Femenino } & \multicolumn{2}{|c|}{ Masculino } & \multicolumn{2}{|c|}{ Chalhuahuacho } & \multicolumn{2}{|c|}{ Progreso } & \multicolumn{2}{|c|}{ Haquira } & & \\
\hline & $\mathbf{n}$ & $(\%)$ & $\mathbf{n}$ & $(\%)$ & $\mathbf{n}$ & $(\%)$ & $\mathbf{n}$ & $(\%)$ & $\mathbf{n}$ & $(\%)$ & $\mathbf{n}$ & $(\%)$ \\
\hline \multicolumn{13}{|l|}{ Arsénico en orina } \\
\hline$\leq 100,0 \mu \mathrm{g} / \mathrm{L}^{*}$ & 255 & $(95,1)$ & 177 & $(95,7)$ & 201 & $(93,9)$ & 146 & $(94,8)$ & 85 & $(100)$ & 432 & $(95,4)$ \\
\hline$>100,0 \mu \mathrm{g} / \mathrm{L}$ & 13 & $(4,9)$ & 8 & $(4,3)$ & 13 & $(6,1)$ & 8 & $(5,2)$ & 0 & $(0,0)$ & 21 & $(4,6)$ \\
\hline \multicolumn{13}{|l|}{ Cadmio en orina } \\
\hline$\leq 1,00 \mu \mathrm{g} / \mathrm{L}^{*}$ & 162 & $(60,4)$ & 92 & $(49,7)$ & 117 & $(54,7)$ & 89 & $(57,8)$ & 48 & $(56,5)$ & 254 & $(56,1)$ \\
\hline$>1,01 \mu \mathrm{g} / \mathrm{L}$ & 106 & $(39,6)$ & 93 & $(50,3)$ & 97 & $(45,3)$ & 65 & $(42,2)$ & 37 & $(43,9)$ & 199 & $(43,9)$ \\
\hline \multicolumn{13}{|l|}{ Colinesterasa sérica } \\
\hline$\leq 4300 \mathrm{U} / \mathrm{mL}$ & 61 & $(22,8)$ & 75 & $(40,5)$ & 57 & $(26,6)$ & 52 & $(27,1)$ & 23 & $(33,8)$ & 132 & $(29,1)$ \\
\hline $4301-10500 \mathrm{U} / \mathrm{mL}$ & 127 & $(47,4)$ & 54 & $(29,2)$ & 74 & $(34,6)$ & 78 & $(35,3)$ & 30 & $(50,6)$ & 182 & $(40,2)$ \\
\hline$>10500 \mathrm{U} / \mathrm{mL}^{*}$ & 80 & $(29,9)$ & 56 & $(30,3)$ & 83 & $(38,8)$ & 24 & $(37,6)$ & 32 & $(15,6)$ & 139 & $(30,7)$ \\
\hline \multicolumn{13}{|l|}{ Mercurio en orina } \\
\hline$\leq 5,00 \mu \mathrm{g} / \mathrm{L}^{*}$ & 264 & $(98,5)$ & 181 & $(97,8)$ & 211 & $(98,6)$ & 153 & $(99,4)$ & 81 & $(95,7)$ & 445 & $(98,2)$ \\
\hline$>5,00 \mu \mathrm{g} / \mathrm{L}$ & 4 & $(1,5)$ & 4 & $(2,2)$ & 3 & $(1,4)$ & 1 & $(0,6)$ & 4 & $(4,7)$ & 8 & $(1,8)$ \\
\hline \multicolumn{13}{|l|}{ Plomo en sangre } \\
\hline$\leq 10,00 \mu \mathrm{g} / \mathrm{dL}^{*}$ & 214 & $(79,9)$ & 129 & $(69,7)$ & 176 & $(82,2)$ & 85 & $(55,2)$ & 82 & $(96,5)$ & 343 & $(75,7)$ \\
\hline $10,01-14,90 \mu \mathrm{g} / \mathrm{dL}$ & 29 & $(10,8)$ & 27 & $(14,6)$ & 20 & $(9,3)$ & 34 & $(22,1)$ & 2 & $(2,4)$ & 56 & $(12,4)$ \\
\hline $14,91-19,90 \mu \mathrm{g} / \mathrm{dL}$ & 18 & $(6,7)$ & 18 & $(9,7)$ & 13 & $(6,1)$ & 22 & $(14,3)$ & 1 & $(1,2)$ & 36 & $(7,9)$ \\
\hline $19,91-44,90 \mu \mathrm{g} / \mathrm{dL}$ & 7 & $(2,6)$ & 11 & $(5,9)$ & 5 & $(2,3)$ & 13 & $(8,4)$ & 0 & $(0,0)$ & 18 & $(4,0)$ \\
\hline Total & 268 & $(59,2)$ & 185 & $(40,8)$ & 214 & $(53,2)$ & 154 & $(34,0)$ & 85 & $(18,8)$ & 453 & $(100)$ \\
\hline
\end{tabular}

*Valores normales 
determinados en las tres regiones geográficas del país ya que entre ellas las prevalencias son diferentes, incluso dentro de los distintos poblados (12). La zona de estudio estaría catalogada como un área de baja endemicidad, lo cual contrasta con estudios realizados en los valles interandinos de Huanta (Ayacucho) ${ }^{(13)}$ y Abancay (Apurímac) ${ }^{(14)}$ que se encuentran en la sierra central-sur del país.

Por otro lado, la ausencia de marcadores serológicos en niños y adolescentes podría ser explicada por la disponibilidad para vacunación de todos los niños menores de un año residentes en áreas de mediana y alta endemicidad desde $1996{ }^{(12) .}$

Con relación a sífilis, los datos de prevalencia no difieren de estudios realizados en población general en otras zonas andinas ${ }^{(15-17)}$. De esta manera, previendo que se incrementen las situaciones de hacinamiento y los riesgos de promiscuidad, tornando vulnerable la población frente a infecciones de transmisión sexual (ITS), se puede inferir un mayor riesgo de transmisión madre-niño. Además de no haberse encontrado ningún caso de VIH, esta situación puede variar por las nuevas costumbres, estilos de vida o escala de valores que la mano de obra foránea traiga consigo como producto de la migración.

La serología positiva para el dosaje de metales pesados en los pobladores, evidencia la presencia de la actividad minera artesanal en la zona (18), la cual responde a una combinación de supervivencia y oportunidad que se da en nuestro país en donde la dicha actividad se ha convertido en un importante generador de empleo para personas que no pueden insertarse en los cada vez más débiles mercados laborales. Sin embargo, según los resultados hallados (cadmio: 43,9\%, plomo: $24,3 \%$, arsénico: $4,6 \%$ y mercurio: $1,8 \%$ ) esta actividad no se está realizando con el debido manejo medioambiental y niveles de seguridad ocupacional que requiere, lo que trae como consecuencia un impacto negativo en el medio ambiente, con alteraciones en los ecosistemas y en la salud humana, como ya ha sucedido en otras áreas del Perú (19).

La determinación de la enzima colinesterasa sérica (butirilcolinesterasa) es una de las pruebas biológicas de mayor valor para la vigilancia y el control de personas expuestas a plaguicidas ${ }^{(20)}$. En este estudio, se encontró que el $29,1 \%$ de la población participante presentó valores de colinesterasa por debajo del rango referencial ${ }^{(21,22)}$. La disminución en la actividad de dicha enzima refleja una síntesis alterada a causa de intoxicación por insecticidas organofosforados. Estos casos son frecuentes y se dan en parte de la población tanto latinoamericana como la que habita en países en desarrollo ya que se dedican a la agricultura y residen en sectores rurales en donde se hace uso elevado de plaguicidas sintéticos ${ }^{(23)}$, los cuales ingresan al organismo por la vía respiratoria, digestiva, conjuntival y dérmica, causando un alto porcentaje de intoxicaciones, que pueden provocar la muerte por falla respiratoria como consecuencia de una combinación del bloqueo del centro respiratorio, broncoespasmo y parálisis de los músculos respiratorios.

Los casos de intoxicaciones humanas son frecuentes, por lo que la vigilancia de su uso debe ser constante por encontrarse ligados no solo a aspectos nutricionales sino también a factores socioeconómicos. Un alto porcentaje de la población de América Latina y El Caribe vive en la pobreza, situación en la que se asocian el infraconsumo, la desnutrición, el alcoholismo, las precarias condiciones de vida, los bajos niveles educacionales, las malas condiciones sanitarias, el hacinamiento, la inestabilidad laboral y la poca participación en los mecanismos de integración social.

Respecto a los niveles encontrados de retardo mental fronterizo y leve en niños menores de 12 años, a pesar de no ser muy altos, se sabe que en países en desarrollo como el nuestro, se debe tener en cuenta las alteraciones en el estado físico y psíquico que podrían ser causadas por el hecho de provenir de ambientes marginales, en donde la pobreza, la escasez de alimentos ${ }^{(24)}$, la deficiente alimentación y las malas condiciones sanitarias pueden influir en que el niño presente menor talla en comparación a otros de su misma edad. Por otro lado, las privaciones sociales, una estimulación insuficiente y el abandono materno-familiar representan un alto riesgo para el desarrollo del infante; en esta investigación se encontró que el $12,5 \%$ de los niños menores de tres años presentó riesgo en su desarrollo psicomotor, situación que debe ser atendida ya que se sabe que un ambiente psicosocial carente de estímulos trae consigo un menor rendimiento y desenvolvimiento por parte de los niños al compararse con aquellos que viven en ambientes estimulantes ${ }^{(25)}$. Por ello, la estimulación disponible en el hogar, sin mediar un programa de estimulación temprana, puede ser suficiente para dar lugar a diferencias en el desarrollo de los niños.

Como parte del actual abordaje de promoción de la salud, la valoración de la salud mental está tomando importancia debido al reconocimiento de su grado de influencia en el desarrollo nacional. Es por ello que la Estrategia Sanitaria Nacional de Salud Mental y Cultura de Paz (ESNSMyCP) busca posicionar a la salud mental como un derecho humano y un componente indispensable para el desarrollo nacional sostenible a través del fortalecimiento y desarrollo del potencial humano en salud mental. En el presente estudio, al evaluar la salud mental en términos de ansiedad y 
depresión, se observó coexistencia de ambos trastornos, encontrándose mayor prevalencia de ansiedad (26\%) frente a depresión (14.3\%), por lo que es necesaria la participación de la ESNSMyCP. Cabe mencionar que estos resultados difieren de los de otras investigaciones realizadas en áreas rurales de Perú ${ }^{(26)}$ y Argentina ${ }^{(29)}$, en donde los trastornos depresivos prevalecieron sobre los de ansiedad (Perú: 46\% y 18\%; Argentina: $24 \%$ y $11 \%$, respectivamente).

Por lo tanto, nuestros resultados muestran que el principal problema que enfrenta este grupo poblacional es la presencia de metales pesados (cadmio y plomo) en su organismo. En segundo lugar, se encuentran los problemas relacionados con enfermedades transmisibles, tales como hepatitis B y sífilis. Un tercer grupo de problemas está en relación a la salud mental de los pobladores, en donde se encontró trastornos de ansiedad y depresivos. Sumado a ello, se registran los casos de riesgo en el desarrollo psicomotor y deficiente coeficiente Intelectual en niños menores de 12 años.

En síntesis, el perfil epidemiológico muestra que, a pesar que la actividad minera aún no se lleva a cabo en la zona de Las Bambas, ya existen indicios de contaminación ambiental, presencia de enfermedades transmisibles, casos de trastornos en la salud mental y alteraciones en el desarrollo psicomotor y coeficiente intelectual. Por tanto, se prevee que la explotación minera podría generar mayor contaminación y un incremento de estos y otros problemas de salud en esta población.

\section{AGRADECIMIENTOS}

A todos los profesionales de salud de los distritos de Haquira, Chalhuahuacho y Progreso por su apoyo en la realización de este estudio. Asimismo, un reconocimiento a la población participante por la valiosa información aportada.

\section{Financiamiento}

Instituto Nacional de Salud.

\section{Conflictos de Interés}

Los autores declaran no tener conflictos de interés en la ejecución y publicación de este artículo.

\section{REFERENCIAS BIBLIOGRÁFICAS}

1. Prolnversión. Las Bambas: un modelo de desarrollo sostenible. Lima: Prolnversión; 2005

2. D \& E. Desarrollo y Ecología SAC. Evaluación ambiental: Proyecto de exploración Las Bambas-Volumen I. Lima: Xstrata Peru; 2004
3. Xstrata Cooper. Proyecto minero Las Bambas. Informe de sostenibilidad 2007. Lima: Xstrata Cooper; 2007.

4. Perú, Instituto Nacional de Estadística e Informática. Perfil de la pobreza por departamentos.2005-2007. Lima: INEl; 2008.

5. Plá MA, Vicente TJ, García FA. Evaluación de riesgos toxicológicos en ecosistemas terrestres. Rev Toxicol 2001;18(3):137-39.

6. Espinoza N, Mariano M, Porlles J, Romero A. Estrategias regionales de gestión ambiental. El caso del proyecto minero Las Bambas de Apurímac. Rev Inv Fac Cienc Admin UNMSM. 2006;9(18):33-9.

7. Christiani D, Durvasula R, Myers J. Occupational health in developing countries: review of research needs. Am J Ind Med. 1990;17(3):393-401.

8. Robaina C, Robaina F. La epidemiología ocupacional en países en desarrollo. Rev Cubana Med Gen Integr 2004;20(2):e6.

9. Organización Panamericana de la Salud. Guías para el control de calidad de la serología para la detección de infección trasmitidas por transfusión (hepatitis B y C, sífilis, $\mathrm{VIH}$ y tripanosomiasis americana). Montevideo: OPS; 1993.

10. Perú, Ministerio de Salud. Tepsi: test de desarrollo psicomotor 2-5 años. Lima: MINSA; 2006.

11. Terman LM, Merril MA. Medida de la inteligencia: Método para el empleo de las pruebas de Stanford-Binet nuevamente revisadas. Madrid: Espas-Calpe; 1970.

12. Cabezas C. Hepatitis virales B y delta: epidemiología y bases para su control. Rev Peru Med Exp Salud Publica. 2007;24(4):378-97.

13. Cabezas C, Gotuzzo E, Escamilla J, Philips I. Prevalencia de marcadores serológicos de hepatitis viral A, B y Delta en escolares aparentemente sanos de Huanta (Perú). Rev Gastroenterol Peru. 1994;14(2):123-34.

14. Indacochea S, Gotuzzo E, De la Fuente J, Phillips I, Whignal S. Elevada prevalencia de marcadores de hepatitis B y Delta en el valle interandino de Abancay. Rev Med Hered. 1991;2(4):168-72.

15. García P, Chávez S, Feringa B, Chiappe M, Li W, Jansen C, et al. Reproductive tract infections in rural women from the highlands, jungle, and coastal regions of Peru. Bull World Health Organ. 2004;82(7):483-91.

16. Perú, Ministerio de Salud. Plan nacional de prevención y control de la transmisión Madre-Niño del VIH y Sífilis 2007 - 2011. Lima: MINSA; 2007.

17. Perú, Ministerio de Salud. Análisis de la situación epidemiológica del VIH/SIDA en el Perú. Bases epidemiológicas para la prevención y control. Lima: DGE/ MINSA; 2006. Serie de análisis de situación y tendencias N. ${ }^{\circ} 16$.

18. Kuramoto J. La minería artesanal e informal en el Perú. Lima: Grupo de Análisis para el Desarrollo (GRADE); 2001.

19. Hurtado J, Gonzales GF, Steenland K. Mercury exposures in informal gold miners and relatives in southern Peru. Int $\mathrm{J}$ Occup Environ Health. 2006;12(4):340-45.

20. Pineda J. Plaguicidas: monitoreo efectivo de la exposición a carbamatos y organosfosforados. Cienc Trab. 2007;9(26):178-81. 
21. Carmona-Fonseca J. Valores de referencia de la actividad de la colinesterasa eritrocitaria según las técnicas de Michel y EQM® en población laboral de Antioquia, Colombia. Rev Panam Salud Publica. 2003;14(5):316-24.

22. Carmona-Fonseca J. Relación entre los niveles de colinesterasa y los grupos sanguíneos $A B O$ y Rh. Acta Med Colomb 2006;31(3):104-12.

23. Ibarra EJ, González A, Díaz H, Jaime A, González RM, Guevara T, et al. Exposición a plaguicidas inhibidores de la colinesterasa en fumigadores de la campaña anti Aedes aegypti en la ciudad de La Habana de enero a marzo de 2002. Rev Cubana Salud Trabajo. 2002;3(1-2):51-54.

24. Perroni M, Schnaas L, Arteaga A, Martínez S, Hernández C, Osorio E. Efecto de la lactancia sobre el desarrollo neuroconductual del niño. Perinatol Reprod Hum. 2003;17(1):20-27.
25. Pando M, Aranda C, Amecua MT, Salazar JP, Torres TM. Estimulación temprana en niños menores de 4 años de familias marginadas. Rev Mex Pediatr. 2004;71(6);273-77.

26. Kendall R, Matos L, Cabra M. Salud mental en el Perú luego de la violencia política. Intervenciones itinerantes. An Fac Med (Lima). 2006;67(2):184-90.

27. Festorazzi A, Rodríguez A, Lotero J. Epidemiologia de los trastornos de ansiedad y depresión en adolescentes de una población rural. Rev Posg Catedr Med. 2008;184:4-9.

Correspondencia: Jonh Astete Cornejo.

Dirección: Las Amapolas 350, Lince, Lima 14.

Correo electrónico: astetemed@gmail.com

\section{Suscríbase en forma electrónica y gratuita a los contenidos de la Revista Peruana de Medicina Experimental y Salud Pública, ingrese a WWW.ins.gob.pe, seleccione el ícono de la revista y envíenos sus datos.}
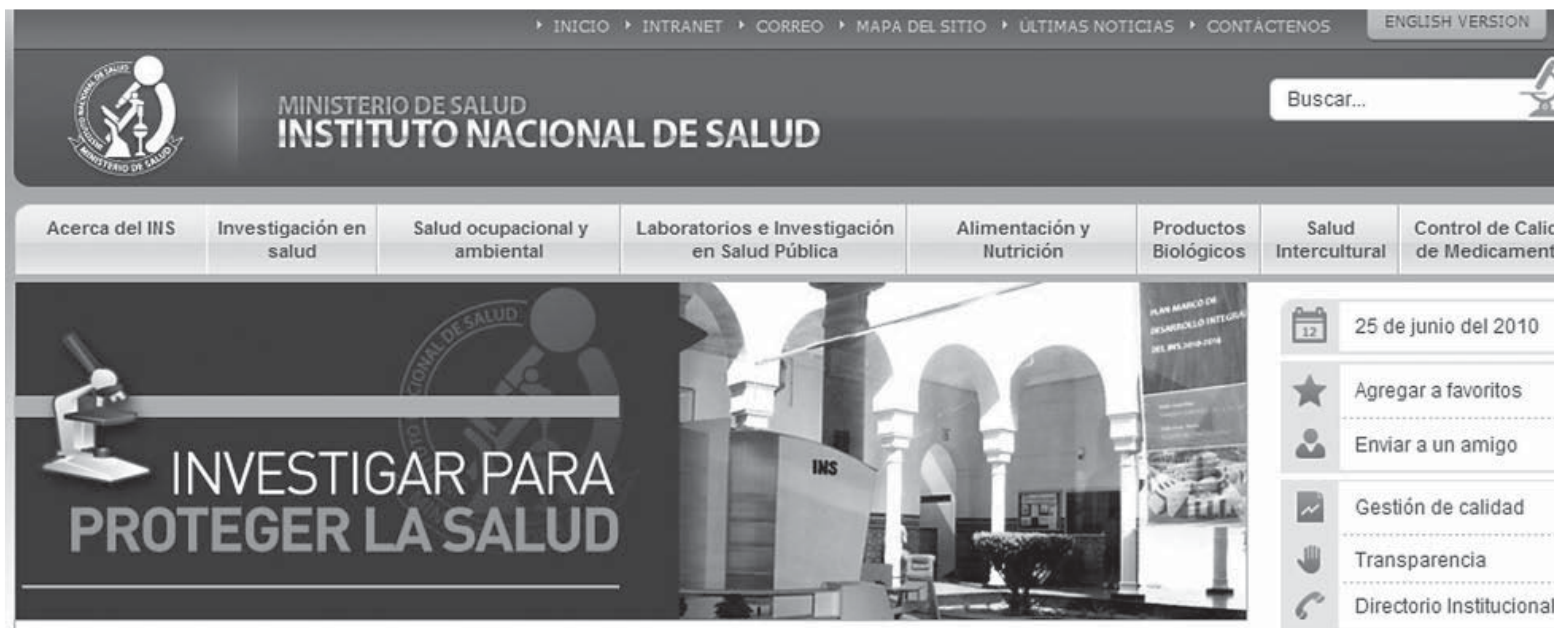

ÚLTIMAS INVESTIGACIONES Y EVIDENCIAS CIENTIFICAS Ver Todas
Programa de Investigación : Reporte de Vigilancia

de Hidatidosis en Zonas Virológica de Influenza y

Endémicas

05-01-2010

Resultados del Programa de

Investigación de HIDATIDOSIS.

Ver detalle

otros Virus Respiratorios

1-05-2010

Ver archivo.

Ver detalle

Verdetile

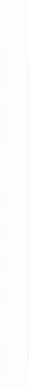

GALERÍA

Ver Todas

Imagen del dia

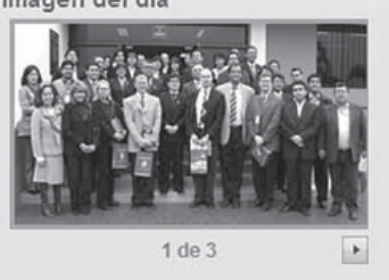

1 de 3

CALENDARIO DE EVENTOS

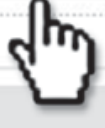

INVESTIGACION

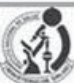

